\title{
Demineralized Bone Matrix-Induced Ectopic Bone Formation in Rats: In Vivo Study with Follow-up by Magnetic Resonance Imaging, Magnetic Resonance Angiography, and Dual-Energy X-Ray Absorptiometry
}

\author{
ED H.M. HARTMAN, M.D., ${ }^{1}$ JEROEN A. PIKKEMAAT, Ph.D., ${ }^{2}$ \\ JACQUES J. VAN ASTEN, M.Sc., JOHAN W.M. VEHOF, M.D., Ph.D., 3 \\ AREND HEERSCHAP, Ph.D., ${ }^{2}$ WIM J.G. OYEN, M.D., Ph.D., ${ }^{4}$ \\ PAUL H.M. SPAUWEN, M.D., Ph.D., ${ }^{1}$ and JOHN A. JANSEN, D.D.S., Ph.D. ${ }^{3}$
}

\begin{abstract}
The aim of this study was to further explore the use of magnetic resonance imaging (MRI), magnetic resonance angiography (MRA), and dual-energy X-ray absorptiometry (DEXA) to assess bone formation and blood circulation in a pedicled bone graft substitute. In 14 Wistar rats, initially 10 weeks old, heterogeneous demineralized femur bone matrix implants were wrapped in pedicled adductor thigh muscle flaps. One rat died after surgery. Subsequently, bone formation and maintenance of blood vessel functionality were evaluated in six rats 6 weeks postimplantation by means of in vivo MRI/MRA and postmortem histomorphometry. The other seven rats were left for 12 weeks, whereafter bone formation was evaluated by in vivo DEXA and postmortem histomorphometry. The results demonstrated that after 6 weeks bone formation was present in four of six animals, quantified as $42( \pm 35) \%$ and $25( \pm 19) \%$ by means of MRI and histomorphometry, respectively. MRA was able to show patency of the pedicles of these four rats only, which suggests that the lack of blood supply in the other two rats is the cause of the failure to form bone. In the 12-week group, histology showed increased bone formation without signs of osteolysis, which was quantified histomorphometrically to be as high as $48( \pm 15) \%$. DEXA failed to show bone formation. It is concluded that in vivo MRI proved to be a reliable method for monitoring ectopic bone formation in a rat model, whereas in vivo DEXA was unable to detect the implants. Furthermore, in vivo MRA proved to be a useful technique for studying the circulation of muscle flaps in this animal model.
\end{abstract}

\section{INTRODUCTION}

$\mathbf{I}$ N RECONSTRUCTIVE BONE SURGERY donor site morbidity is a consequence of the use of autogenous grafts. ${ }^{1}$ Tissue engineering is a promising new technique that can help to minimize this donor site morbidity by creating a bone graft substitute (BGS). As part of our efforts to produce BGSs, we explored in a previous study the feasibility of using magnetic resonance imaging (MRI) as a method to assess bone formation in tissue-engineered bone constructs. ${ }^{2}$ In this pilot study, demineralized bone matrix (DBM) implants were inserted subcutaneously in

\footnotetext{
${ }^{1}$ Department of Plastic Surgery, University Medical Center Nijmegen, University of Nijmegen, Nijmegen, The Netherlands.

${ }^{2}$ Department of Radiology, University Medical Center Nijmegen, University of Nijmegen, Nijmegen, The Netherlands.

${ }^{3}$ Department of Biomaterials, University Medical Center Nijmegen, University of Nijmegen, Nijmegen, The Netherlands.

${ }^{4}$ Department of Nuclear Medicine, University Medical Center Nijmegen, University of Nijmegen, Nijmegen, The Netherlands.
} 
a single rat. Subsequently, at 1, 3, 5, and 7 weeks postimplantation, MRI was done to monitor bone formation in the implants. The results showed that (1) in vivo MRI is suitable to check bone formation noninvasively; and (2) MRI results match well with histology results obtained at 7 weeks. However, in this preliminary study, no attempt was made to quantify the MRI results and to compare the quantitative MRI data with standard histomorphometry. Therefore, the first aim of the current study is to continue and expand our in vivo MRI studies for boneengineering applications.

Besides the use of MRI to produce pictures of body tissue, MRI technology can also be utilized to detect blood vessels. This so-called MR angiography (MRA) provides detailed information about the functionality of blood vessels without using any contrast material. At this point, it appears appropriate to emphasize that the final goal in our bone-engineering studies is to create a prefabricated and preshaped bone graft consisting of an osteoinductive scaffold combined with a vascularized muscle flap, which can be transplanted microsurgically to the receptor area. Maintenance of blood flow is of major importance for the final success of such free flap constructs. Consequently, the second aim of the present study was to determine whether MRA is indeed a useful method to determine the functionality of the supplying blood vessel in such pedicled bone graft substitutes.

A third aim of the current study was also to evaluate another technique for the assessment of bone formation, that is, dual energy X-ray absorptiometry (DEXA). This is a fast, inexpensive, and reproducible method to examine bone mineral content and density in clinical practice. ${ }^{3,4}$ In addition, the efficacy of this technique for the measurement of bone formation in porous implants has been reported. ${ }^{5,6}$

Considering these three aims, we created, in rats, pedicled muscle flaps that were wrapped around DBM implants. Two groups of rats were studied in our study: a so-called early group (6 weeks) and a late group (12 weeks). In vivo MRI and postmortem histology were used to evaluate bone formation in the early group. Also, MRA was performed to determine the functionality of the blood vessels in the pedicled DBM implant. For the late group, in vivo DEXA and postmortem histology were used for bone formation analysis.

\section{MATERIALS AND METHODS}

\section{Implants}

The femora of 7 male Wistar donor rats (10 weeks old) were used to prepare 14 DBM implants. From each femur the bone marrow was removed and $1-\mathrm{cm}$ sections of the diaphyses were made. These were immediately chilled to $0^{\circ} \mathrm{C}$ and then decalcified in hydrochloric acid $(\mathrm{HCl})(1 \mathrm{~g}$ of bone per $100 \mathrm{~mL}$ of $0.6 \mathrm{~N} \mathrm{HCl})$ under constant stirring for $48 \mathrm{~h}$ at a temperature of $2^{\circ} \mathrm{C}$. The $\mathrm{HCl}$ was refreshed after $24 \mathrm{~h}$. After dilution of the acid with $0.15 \mathrm{~N} \mathrm{NaCl}$, the implants were lyophilized and stored at $-20^{\circ} \mathrm{C}$. The average weight of the DBM implants was $19 \pm 4 \mathrm{mg}$.

\section{Implantation procedure}

Fourteen male Wistar rats (WU, 10 weeks of age; average weight, $280 \mathrm{~g}$ ) were used in the study. Before surgery, the animals were anesthetized with a mixture of $50 \%$ oxygen with $50 \%$ nitrous oxide and $1.2 \%$ isoflurane (1-chloro-2,2,2-trifluoroethyl-difluoromethyl ether). For the insertion of implants, the animals were immobilized and placed in a supine position. During the implantation procedure the core temperature was registered with a rectal probe and maintained with a variable-temperature circulating-water bed. The skin of the animals was shaved, washed, and disinfected with povidone-iodine. After incising the skin on the medial side of the hind limb, the saphenous vessels were distally ligated, just above the point where the vessels split in two or three branches. The anterior and posterior gracilis muscles were elevated from distal to proximal, preserving the branches of the saphenous vessels to these muscles. Proximally from the muscles, the saphenous vessels originate from the femoral vessels. All other branches from the femoral vessels were ligated and cut to provide optimal blood flow to the muscle flap. Each animal received one DBM implant, which was wrapped in the adductor thigh muscle flap. ${ }^{7}$ The flap was closed with nonabsorbable monofilament 7-0 sutures (Nylon). Around this muscle flap, a $1.5 \times 1.0 \mathrm{~cm}$ silicone sheet with a thickness of $0.5 \mathrm{~mm}$ was wrapped to prevent vascular ingrowth from the surrounding tissues. Consequently, the flap was vascularized solely from its pedicle. At the end of the operation, the skin was closed with stainless steel staples.

After surgery, the animals were randomly assigned to two groups. The early group $(n=7)$ was maintained for 6 weeks and the late group $(n=7)$ was maintained for 12 weeks. At 6 weeks after implantation, MRI/MRA was performed on all rats of the early group. Thereafter, the animals were killed by an overdose of ether anesthesia and implants together with surrounding tissues were retrieved for histological evaluation. Twelve weeks after implantation, DEXA scans were obtained for all rats belonging to the late group. Subsequently, these animals were also killed and implants plus surrounding tissue retrieved for histological analysis.

The animal protocol used was approved by the animal care ethics committee of the University of Nijmegen. The animal experiments were performed according to guidelines for animal experiments for scientific research of the Dutch government. (Experiments on Animals Act, 1977). 


\section{Evaluation techniques}

Magnetic resonance imaging/magnetic resonance angiography. All MRI and MRA experiments were carried out on a Survey Medical Imaging Systems (Guildford, UK) 7-T MRI scanner with a 200-mm bore size dedicated for small test animals. Animals were placed into the magnet, face down, on top of a 22-mm radiofrequency (RF) surface coil covering one DBM implant at a time. During the MRI/MRA investigation, the rat was anesthetized as indicated in the implantation procedures, the respiration rate was continuously monitored, and the core temperature was maintained as indicated in the implantation procedures. For the MRI experiment we used a gradient echo sequence $(\mathrm{TE}=6 \mathrm{~ms}$; $\mathrm{TR}=1000 \mathrm{~ms}$; flip angle $=60^{\circ}$; field of view $=55 \mathrm{~mm}$; matrix size $=512 \times$ 512 data points). The images show mainly proton density weighting. As shown in a previous article, areas with newly formed bone will appear dark (calcification) in proton density-weighted images in contrast to nonresponding DBM, which appear white (no calcification). ${ }^{2}$ After a trial scan to establish the orientation of the DBM implants, 24 parallel slices (thickness, $0.7 \mathrm{~mm}$; separation, $1.0 \mathrm{~mm}$ ) were acquired perpendicular to the long axis through the DBM. As the length of the DBM implants was $10 \mathrm{~mm}$, each DBM was visible in 10 contiguous slices. The percentage of calcification in each slice was calculated. Thereafter, the data for the separate slices were averaged, which resulted in a volume percentage calcification of the total implant volume, for example, $45 \%$ calcification means $45 \%$ of the total implant volume. Finally, the same slices were imaged with an MRA sequence $(\mathrm{TE}=6 \mathrm{~ms}$; $\mathrm{TR}=200 \mathrm{~ms}$; flip angle $=$ $90^{\circ}$ ) as well. In these images, regions with large flow (functional blood vessels) appear white, and other areas appear dark. Thus the MRA images can be used to demonstrate the integrity of the vascular pedicle.

DEXA. DEXA scans were performed on a QDR 1000 scanner (Hologic, Waltham, MA). The rats were anesthetized by halothane-nitrous oxide-oxygen inhalation and placed prone on the scanner. The hind limbs were fixed to prevent projection of the femur over the implant. After acquiring a scan of the hind limb area, the femora and the DBM implants were identified. It was attempted to quantify the bone mineral content of the DBM by positioning regions of interest over the location of the implant and over the surrounding tissues.

Histological analysis. Specimens were retrieved for histological analysis: they were fixed and stored in phosphate-buffered $4 \%$ formalin solution ( $\mathrm{pH}$ 7.4). The tissue blocks were then dehydrated in a graded series of ethanol and embedded in methyl-methacrylate (MMA). Subsequently, $10-\mu \mathrm{m}$ sections of the MMA-embedded sam- ples were made with a modified sawing microtome technique. ${ }^{8}$ At least three transverse sections were made at five different levels in each implant. These levels corresponded with the transverse slices of the MRI evaluation. The sections were stained with methylene blue and basic fuchsin. To evaluate the tissue response to the implants, both histological and histomorphometric analysis was performed. The histological evaluation consisted of a complete description of the observed thin sections. A computer-based image analysis system (Qwin Pro, version 2.3; Leica, Wetzlar, Germany) was used for histomorphometric analysis. Two parameters were assessed: (1) the surface area of the newly formed bone and (2) the surface area of the DBM implant. In this way relative bone formation could be measured. All histomorphometric measurements were performed in the five different transverse sections of each implant, which were averaged to obtain the final result.

\section{Statistical procedure}

Results were expressed as means \pm 1 standard deviation (SD). To test the correlation between MRI and histomorphometry a linear correlation analysis was done. Data of the histomorphometrical analysis were compared with an unpaired $t$ test. The test was performed at the 95\% confidence level. The $t$ test assumes that the data are sampled from populations that follow Gaussian distributions. This assumption was tested according to the method of Kolmogorov and Smirnov. All calculations were performed in GraphPad Instat version 3.05 for Windows 95/NT (GraphPad Software, San Diego, CA).

\section{RESULTS}

\section{Clinical observations}

One of the rats of the early group died after surgery. Therefore, this group ultimately consisted of six rats. All rats showed undisturbed wound healing without any clinical signs of inflammation at the surgical sites during the various implantation times.

\section{Early group}

MRI/MRA. Six implants were evaluated by MRI and MRA. The quantitative data for these six implants are presented in Fig. 1. In four implants clear bone formation was observed on MRI, all of them having an intact vascular pedicle on MRA (Fig. 2). Two implants showed only minimal bone formation, while the patency of the pedicle could not be proven by MRA. The mean relative bone formation for all implants was $42 \pm 35 \%$.

Descriptive light microscopy. Light microscopy confirmed the MRI results, that is, bone formation was seen 


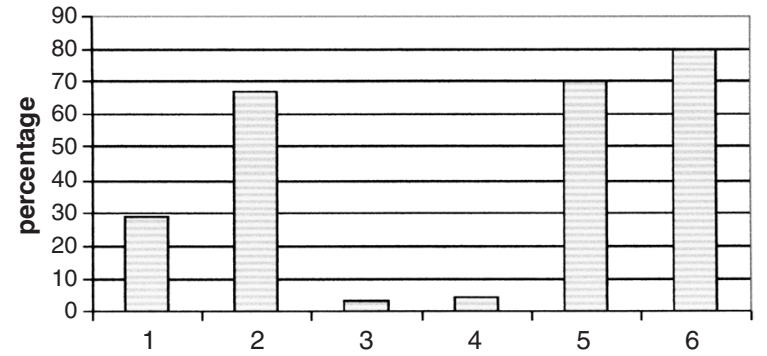

FIG. 1. Quantitative MRI data for implants in the early group. Four implants $(1,2,5$, and 6$)$ show marked bone formation; two implants show almost no bone formation.

in only four of the six specimens. Bone formation was characterized by the occurrence of mineral deposits inside the DBM material. Bone was deposited in islands and was not present as a continuous phase in the DBM. Bone formation could be associated with the presence of osteoblasts, osteoid, and osteocytes (Fig. 3). Inside the bony islands, cavities were present that were filled with bone marrow-like tissue. Bone formation did not expand outside the original geometry of the DBM specimens. At the outer surface, the specimens were surrounded by a fibrous tissue capsule. No inflammatory response inside the capsule or at the capsule-DBM interface was observed.

Histomorphometry. Unfortunately, one specimen could not be used for histomorphometric evaluation, because the available sections were of insufficient quality to allow reliable quantification of the relative bone formation. The results of the other five measurements are depicted in Fig. 4. Three implants showed bone formation of $25 \%$ or more of their total surface area. The other two implants had bone formation of less than $10 \%$. The mean relative value of bone formation for all implants was $25 \pm 19 \%$.

Comparison of MRI and histomorphometry data. To determine the existence of a relationship between MRI and histomorphometric evaluation, a linear correlation analysis was done. The calculated correlation coefficient

\section{MRI}

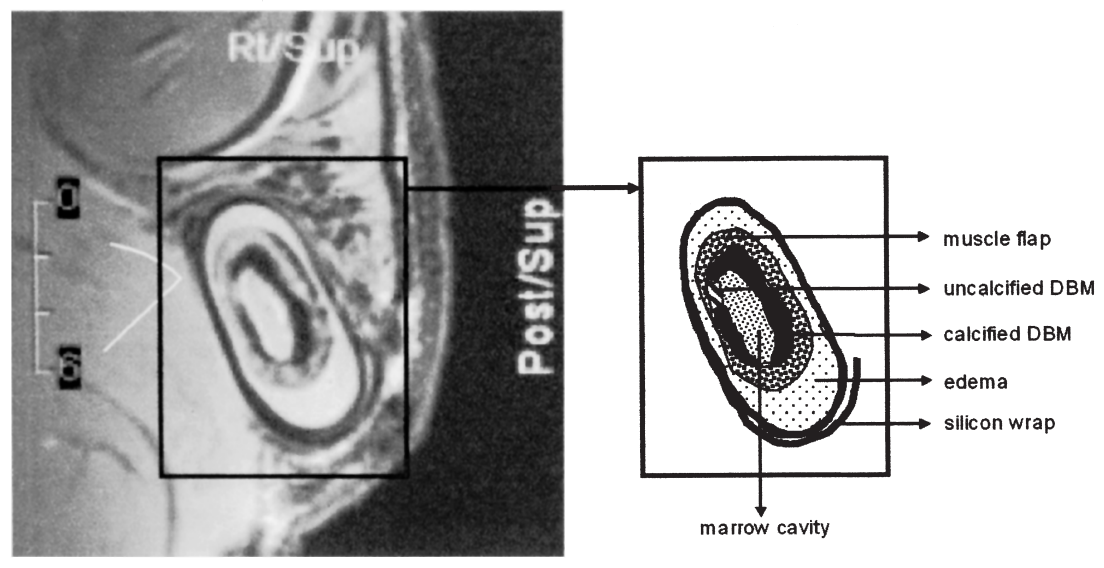

\section{MRA}

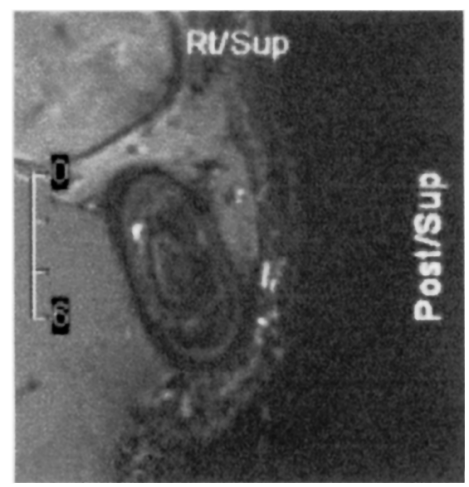

FIG. 2. MRI and MRA of DBM implant. Top (MRI): Bone formation is visible as a dark ring. A silicone sheet is clearly visible (caret). Bottom (MRA): The white spot represents flow in the vascular pedicle of the flap. 

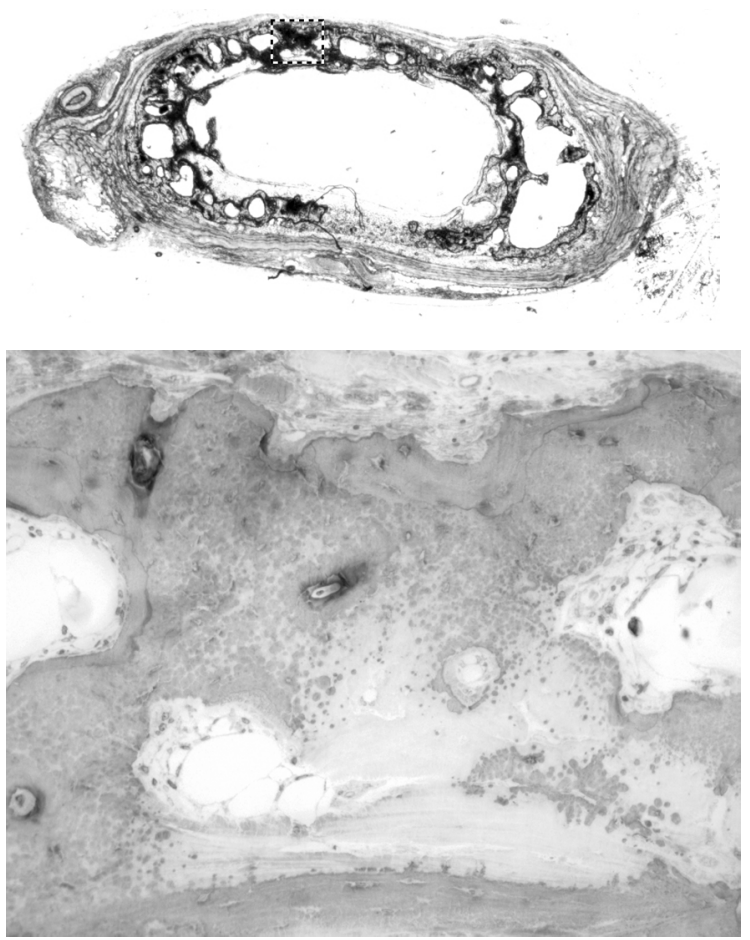

FIG. 3. Histology of an early group specimen: Ectopic bone formation at 6 weeks (original magnification, $\times 1.6$; enlargement, $\times 20)$.

$r$ was 0.9395 and the coefficient of determination $\left(r^{2}\right)$ was 0.8827 . This indicates the existence of a strong correlation.

\section{Late group}

DEXA. The autologous rat femurs were well visualized. However, the density of the implants was hardly distinguishable from the soft tissues. At best, the implants were only faintly visualized, indicating a low bone mineral content. Consequently, region-of-interest quantification did not yield any reproducible results, because it was impossible to reliably discriminate the implant from the surrounding soft tissues.

Descriptive light microscopy. Histological analysis seemed contradictory to the DEXA observations. In five of the DBM specimens, extensive bone formation was observed. Bone formation was again characterized by the presence of osteoblasts, osteoid, and osteocytes in a mineralized matrix. Also, inside the deposited bone, bone marrow-like tissue was observed. On occasion, bone formation extended throughout the DBM and at some sites filled the DBM almost completely. The newly deposited bone never expanded in the original medullar cavity of the DBM or outside the DBM material. In the other two specimens, bone formation could also be observed. How- ever, the amount of bone was clearly less. All DBM specimens maintained their original shape. No sign of DBM degradation was seen. All specimens were further surrounded by a fibrous capsule without the presence of inflammatory cells.

Histomorphometry. Bone formation data of the seven DBM implants of the late group are depicted in Fig. 5. Five implants showed bone formation of $45 \%$ or more of their total surface area. The other two implants revealed a much lower bone formation percentage (less than 25\%). The mean bone formation percentage for all implants was $48 \pm 15 \%$.

\section{Comparison of early and late group data}

Comparison of the histomorphometric data for bone formation between the early and late groups reveals that significantly more bone is formed in the late DBM group $(p=0.0463)$. Additional statistical testing confirmed that the two SD's of the early and late group did not differ significantly from each other $(p=0.3011)$. Also, the data were shown to be sampled from Gaussian distributions $(p>0.10)$.

\section{DISCUSSION}

The current study was an expanded follow-up analysis of an earlier performed pilot experiment and aimed at the use of MRI and DEXA to monitor the progress of bone development in pedicled bone graft substitutes. ${ }^{2}$ In addition, MRA was used to study blood circulation in the created muscle flaps. Bone formation was evaluated in DBM implants, wrapped in pedicled muscle flaps and inserted in rats. MRI and DEXA results were always compared with histomorphometric measurements. Unfortunately, MRI/MRA and DEXA could not be performed on the same animals because of organizational and regula-

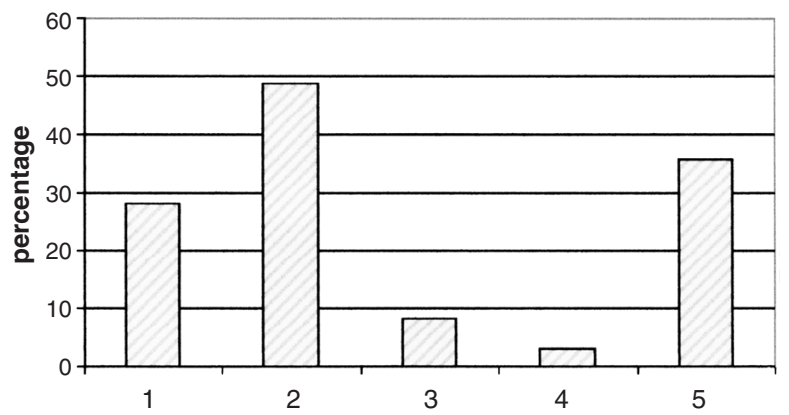

FIG. 4. Histomorphometric analysis of bone formation in early group. Three implants (1, 2, and 5) show marked bone formation; two implants show almost no bone formation. 


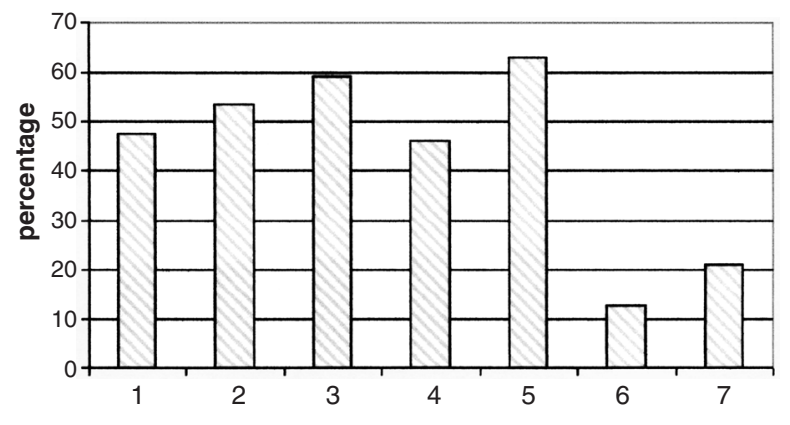

FIG. 5. Histomorphometric analysis of bone formation in late group. Five implants (1, 2, 3, 4, and 5) show marked bone formation; two implants show no marked bone formation (less than $20 \%)$.

tory affairs. The MRI equipment used was available at the animal facility. DEXA equipment was available at the hospital and was used for patient diagnosis. As a consequence, DEXA measurements had to be done outside regular working hours. In addition, MRI for experimental animals is still a costly and time-elaborative technique. Therefore, we decided to create two separate groups and to correlate the obtained data to histomorphometric measurements.

Considering the use of MRI for the analysis of bone development, the major advantage of MRI, compared with the "gold standard" of histology, is the ability to perform in vivo longitudinal studies. Interanimal variation, which cannot be avoided in histological studies, can be prevented in this way. On the other hand, disadvantages of MRI involve potential unavailability of equipment, costs, as well as the necessity for anesthesia with the inherent risk of animal death. Comparison of the available MRI with histological data reveals the existence of a strong relationship between both methods. This confirms that MRI is of sufficient accuracy for comparative and sequential bone formation studies. Consequently, MRI can be a competitive technique for the long-term follow-up evaluation of bone graft substitutes, especially when larger and less elaborate equipment becomes available. Despite this favorable outcome, we must note that MRI and histomorphometry do not indicate exactly the same amounts of bone formation. An explanation can be found in the number of slices and the bone quantification technique used. For MRI, 10 slices were evaluated, representing the total implant. However, the imaging of these relatively thick slices is still a two-dimensional representation of a threedimensional subject, which can result in over- or underestimation of the amount of bone formation. On the other hand, for the histomorphometric analysis only a limited number of sections of a complete specimen can be analyzed, which can also result in over- or underestimation.

Besides MRI, longitudinal studies can also be performed with DEXA scans. As in MRI, the animals must be anesthetized for each investigation. However, the investigation itself is generally fast, inexpensive, and widely available. We chose to use DEXA for the late group and to compare it with the gold standard of histology and histomorphometry. However, the bone mineral content was so low in comparison with surrounding soft tissue density that the graft could hardly be identified. Consequently, DEXA is unsuitable to reliably assess small variations in mineralization, which makes this technique unsuitable for evaluation of bone tissue regeneration in the current experimental design in small laboratory animals. However, given the procedure's highly reproducible evaluation of bone density and bone mineral content in a clinical setting, DEXA may be more suitable when larger animal models and larger bone constructs are used.

Bone inductive activity of demineralized bone preparations is determined by the amount and activity of bone morphogenetic proteins (BMPs) present in the material. Increased bone formation is observed at higher concentrations of BMP. ${ }^{9-12}$ These growth factors are present in cortical bone, but only in minute amounts, approximately 1-2 $\mu \mathrm{g}$ of BMP per kilogram of cortical bone. ${ }^{13}$ The amount of BMPs may vary widely depending on source, ${ }^{14}$ preparative technique, ${ }^{15}$ and sterilization method used. ${ }^{16}$ The quantity of bone formation is also related to the surface area of exposed matrix. ${ }^{17,18}$ Therefore, in most studies DBM is used as particulate and not as a massive implant. ${ }^{19,20}$ Such DBM particles were reported to generate $58 \pm 25 \%$ of bone in a rat craniotomy defect after 3 weeks of implantation. ${ }^{10}$ In the present study, we used DBM as bulk 1-cm sections of a femur. Such DBM implants have a smaller surface area than powder DBM. Nevertheless, bone formation after 6 and 12 weeks proved to be, respectively, $25 \pm 19 \%$ and $48 \pm 15 \%$. Concerning the maintenance of deposited bone over time, we found that bone formation did not stop at 6 weeks, but instead continued so that after 12 weeks the amount of developed bone is significantly higher than at 6 weeks. No signs of osteolysis could be detected. This corroborates with the findings of Viljanen et al., who also described a time-related increase in new bone in a rat latissimus dorsi flap model with DBM, using radiomorphometry to quantify bone formation. ${ }^{21}$ They reported a relative increase in radiographic reference, from 55 to $80 \%$ of rat normal bone average intensity at 10,21 , and 35 days.

In addition, we observed that two of the 6-week samples showed almost no bone formation (less than 10\%). MRA suggests that this is evidently due to a lack of blood supply. This can be due to either kinking of the vascular pedicle with subsequent lack of circulation preventing formation of bone, or compression of the flap by the silicone envelope. Postoperative edema of the muscle flap can result in a relative tightness of this silicone envelop with flap failure as a result. Viljanen et al. already warned 
of this complication, although in their series of 12 DBM implants in a rat latissimus dorsi muscle flap model, almost complete ossification was found in all implants. ${ }^{21}$

Although MRA was found to be a suitable technique to evaluate the maintenance of circulation in the supplying blood vessel in pedicled BGSs, we cannot exclude that the lack of bone formation could also have been caused by the known and marked variation in the boneinducing capacity of DBM. ${ }^{21-25}$ Evidently, the processing technique is important for the effectiveness of DBM and should therefore be standardized. ${ }^{26}$ Despite our efforts to be as accurate as possible, some variation in the processing of the samples could still have occurred. In this respect, we must emphasize that DBM material must be bioassayed before use in order to prevent the variation in osteoinductive effect. ${ }^{27}$

\section{CONCLUSION}

On the basis of our observations, we conclude that in vivo MRI proved to be a reliable method for monitoring ectopic bone formation in a rat model, whereas in vivo DEXA was unable to detect the implants. However, because MRI is time consuming and expensive, it is currently still not suited to replace histology and histomorphometry. Furthermore, MRA proved to be a useful technique for studying the circulation of muscle flaps in this animal model.

\section{REFERENCES}

1. Hartman, E.H.M., Spauwen, P.H.M., and Jansen, J.A. Review of donor site complications in vascularised bone flap surgery. J. Invest. Surg. 15, 185, 2002.

2. Hartman, E.H.M., Pikkemaat, J.A., Vehof, J.W.M., Heerschap, A., Jansen, J.A., and Spauwen, P.H.M. In vivo MRI explorative study on ectopic bone formation in the rat. Tissue Eng. 8, 1029, 2002.

3. Corten, F.G., Caulier, H., van der Waerden, J.P., Kalk, W., Corstens, F.H., and Jansen, J.A. Assessment of bone surrounding implants in goats: Ex vivo measurements by dual X-ray absorptiometry. Biomaterials 18, 495, 1997.

4. Nagy, T.R., Prince, C.W., and Li, J. Validation of peripheral dual-energy X-ray absorptiometry for the measurement of bone mineral in intact and excised long bones of rats. J. Bone Miner. Res. 16, 1682, 2001.

5. Mosheiff, R., Klein, B.Y., Leichter, I., Chaimsky, G., Nyska, A., Peyser, A., and Segal, D. Use of dual energy X-ray absorptiometry (DXA) to follow mineral content changes in small ceramic implants in rats. Biomaterials $\mathbf{3}$, 462, 1991.

6. Kilgus, D.J., Shimoaoka, E.E., Tipton, J.S., and Eberle, R.W. Dual-energy X-ray absorptiometry measurement of bone mineral density around porous-coated cementless femoral implants. J. Bone Joint Surg. Br. 75, 279, 1993.
7. Khouri, R.K., Koudsi, B., and Reddi, H. Tissue transformation into bone in vivo: A potential practical application. JAMA 266, 1953, 1991.

8. van der Lubbe, H.B., Klein, C.P., and de Groot, K. A simple method for preparing thin $(10 \mu \mathrm{m})$ histological sections of undecalcified plastic embedded bone with implants. Stain Technol. 63, 171, 1988.

9. Kusumoto, K., Bessho, K., Fujimura, K., Akioka, J., Ogawa, Y., and Iizuka, T. Prefabricated muscle flap including bone induced by recombinant human bone morphogenetic protein-2: An experimental study of ectopic osteoinduction in a rat latissimus dorsi muscle flap. Br. J. Plast. Surg. 51, 275, 1998.

10. Marden, L.J., Hollinger, J.O., Chaudhari, A., Turek, T., Schaub, R.G., and Ron, E. Recombinant human bone morphogenetic protein-2 is superior to demineralized bone matrix in repairing craniotomy defects in rats. J. Biomed. Mater. Res. 28, 1127, 1994.

11. Wang, E.A., Rosen, V., D’Alessandro, J.S., et al. Recombinant human bone morphogenetic protein induces bone formation. Proc. Natl. Acad. Sci. U.S.A. 87, 2220, 1990.

12. Volek-Smith, H., and Urist, M.R. Recombinant human bone morphogenetic protein (rhBMP) induced heterotopic bone development in vivo and in vitro. Proc. Soc. Exp. Biol. Med. 211, 265, 1996.

13. Tuominen, T. Native Bovine Bone Morphogenetic Protein in the Healing of Segmental Long Bone Defects. Oulu, Finland: University of Oulu, Finland, 2001.

14. Syftestad, G.T., and Urist, M.R. Bone aging. Clin. Orthop. 162, 288, 1982.

15. Syftestad, G., and Urist, M.R. Degradation of bone matrix morphogenetic activity by pulverization. Clin. Orthop. 141, 281, 1979.

16. Urist, M.R., and Hernandez, A. Excitation transfer in bone: Deleterious effects of cobalt 60 radiation-sterilization of bank bone. Arch. Surg. 109, 586, 1974.

17. Sampath, T.K., and Reddi, A.H. Importance of geometry of the extracellular matrix in endochondral bone differentiation. J. Cell Biol. 98, 2192, 1984.

18. Pajamaki, K.J.J., and Viljanen, V.V. Demineralized bone matrix inductive graft. In: Lindholm TS, ed. Bone Morphogenetic Proteins: Biology, Biochemistry and Reconstructive Surgery. Georgetown, TX: R.G. Landes, 1996, pp. 201-205.

19. Nishimoto, S.K., Chang, C.H., Gendler, E., Stryker, W.F., and Nimni, M.E. The effect of aging on bone formation in rats: Biochemical and histological evidence for decreased bone formation capacity. Calcif. Tissue Int. 37, 617, 1985.

20. Hollinger, J.O., Mark, D.E., Goco, P., Quigley, N., Desverreaux, R.W., and Bach, D.E. A comparison of four particulate bone derivatives. Clin. Orthop. 267, 255, 1991.

21. Viljanen, V.V., Gao, T.J., and Lindholm, T.S. Producing vascularized bone by heterotopic bone induction and guided tissue regeneration: A silicone membrane-isolated latissimus dorsi island flap in a rat model. J. Reconstr. Microsurg. 13, 207, 1997.

22. Guizzardi, S., Di Silvestre, M., Scandroglio, R., Ruggeri, A., and Savini, R. Implants of heterologous demineralized bone matrix for induction of posterior spinal fusion in rats. Spine 17, 701, 1992. 
23. Hopp, S.G., Dahners, L.E., and Gilbert, J.A. A study of the mechanical strength of long bone defects treated with various bone autograft substitutes: An experimental investigation in the rabbit. J. Orthop. Res. 7, 579, 1989.

24. Oikarinen, J. Experimental spinal fusion with decalcified bone matrix and deep-frozen allogeneic bone in rabbits. Clin. Orthop. 162, 210, 1982.

25. Schwarz, N., Schlag, G., Thurnher, M., Eschberger, J., Dinges, H.P., and Redl, H. Fresh autogeneic, frozen allogeneic, and decalcified allogeneic bone grafts in dogs. J. Bone Joint Surg. Br. 73, 787, 1991.

26. Russell, J.L., and Block, J.E. Clinical utility of demineralized bone matrix for osseous defects, arthrodesis, and reconstruction: Impact of processing techniques and study methodology. Orthopedics 22, 524 (quiz, 532-533), 1999.
27. Wilkins, R.M., Kelly, C.M., and Giusti, D.E. Bioassayed demineralized bone matrix and calcium sulfate: Use in bone-grafting procedures. Ann. Chir. Gynaecol. 88, 180, 1999.

Address reprint requests to:

Ed H.M. Hartman, M.D.

University Medical Center Nijmegen

305 Department of Plastic Surgery

P.O. Box 9101

6500 HB Nijmegen, The Netherlands

E-mail: hartman@nvpc.nl 